\title{
Réflexions autour de la définition du champ de l'anglistique de spécialité : le cas de l'Europe du Nord
}

John Humbley

\section{OpenEdition}

Journals

Édition électronique

URL : http://journals.openedition.org/asp/491

DOI : 10.4000/asp.491

ISBN : 978-2-8218-0404-3

ISSN : 2108-6354

\section{Éditeur}

Groupe d'étude et de recherche en anglais de spécialité

\section{Édition imprimée}

Date de publication : 1 décembre 2007

Pagination : $53-70$

ISSN : 1246-8185

\section{Référence électronique}

John Humbley, «Réflexions autour de la définition du champ de l'anglistique de spécialité : le cas de l'Europe du Nord », ASp [En ligne], 51-52 | 2007, mis en ligne le 01 décembre 2010, consulté le 22 mars 2021. URL : http://journals.openedition.org/asp/491 ; DOI : https://doi.org/10.4000/asp.491

Ce document a été généré automatiquement le 22 mars 2021.

Tous droits réservés 


\title{
Réflexions autour de la définition du champ de l'anglistique de spécialité : le cas de l'Europe du Nord
}

\author{
John Humbley
}

\section{Introduction}

1 Tous les deux ans, un colloque intitulé European LSP Symposium réunit plusieurs centaines de chercheurs et de pédagogues de tout le continent, mais en particulier des pays du Nord. C'est lors de ces rencontres que l'on peut apprécier, dans les thèmes évoqués et la manière de les traiter, les fortes différences qui subsistent entre les traditions intellectuelles des pays représentés. La dernière en date est celle de Bergame en 2005 ; malgré le cadre latin, le choix des sujets reflète surtout le point de vue des chercheurs du Nord et couvre un vaste éventail, dont l'étendue a surpris au moins un observateur anglo-saxon, Philip Shaw $(2005)^{1}$, qui s'est interrogé sur ces différences ${ }^{2}$. L'opposition entre le pragmatisme des uns et la théorisation des autres relève sans doute de la caricature, mais les différences entre les approches germaniques et celles du reste du monde sont profondes et, à nos yeux, méritent qu'on s'y intéresse.

2 À un moment où les anglicistes français s'interrogent sur l'organisation de leurs activités de recherche, il peut être utile de passer en revue certaines des caractéristiques des études de langues de spécialité (LSP) dans les pays de langue allemande. C'est d'ailleurs le conseil donné par Pierre Cotte lors d'une table ronde consacrée au colloque de la SAES à Nantes en 2006 : il s'agit de s'inspirer des sujets et des méthodes de recherche des pays voisins. L'insistance sur le rôle de l'érudition dans la recherche lui semblait particulièrement digne d'intérêt. Or, le reproche que l'on entend le plus souvent en France concernant les études des langues de spécialité est le manque d'ambition théorique. Même si cette opinion ne correspond pas vraiment à la 
réalité, on peut avoir intérêt à examiner comment d'autres chercheurs en langues de spécialité intègrent théorie et érudition dans leur démarche intellectuelle.

3 C'est lors de la fondation du Centre de terminologie et de néologie (CTN) en 1987 que l'auteur s'est rendu compte de la richesse de la recherche germanique dans ces domaines. Cette petite équipe, placée au sein de l'Institut national de la langue française, était chargée de développer une recherche en terminologie, de participer à deux revues (Terminologies nouvelles et La Banque des mots) et de constituer un centre de documentation. Ces trois missions ont mis l'équipe en contact avec les pays francophones, qui développaient des activités dans le cadre de l'aménagement linguistique, mais plus encore avec ceux de langue allemande, où les études de terminologie se plaçaient - et se placent encore - résolument dans le cadre des langues de spécialité, elles-mêmes situées dans le domaine de la linguistique. Depuis cette première rencontre, nous avons la conviction que les recherches en terminologie, comme en langue de spécialité plus généralement, ne peuvent se faire sans référence à un cadre théorique faisant appel à une méthodologie systématique, dont on trouve des exemples significatifs dans les pays germaniques.

Cette contribution est conçue par ailleurs comme une occasion de renouer avec la politique d'ouverture du GERAS : on pense en particulier aux colloques consacrés aux comparaisons entre l'anglais et le français de spécialité (ASp 15/18) et aux thèmes transversaux, comme celui englobant traductologie et terminologie (ASp 19/20). On pense aussi aux communications présentées par les membres du GERAS aux colloques de LSP, à commencer par le troisième Symposium européen, que Michel Perrin a organisé en 1983 à Bordeaux (Perrin 1983), ou à celle que ce dernier a prononcée à Vienne en 1995 (Perrin 1995).

5 Pour les besoins de cet article, les aires culturelles couvertes comportent non seulement les pays germanophones (Allemagne, Autriche, Suisse), mais aussi ceux où l'allemand joue ou a joué un rôle de langue véhiculaire: dans le passé en Tchécoslovaquie (l'influence de l'école de Prague est particulièrement importante dans l'histoire du développement des études de langues de spécialité en Allemagne), et encore de nos jours en Scandinavie, au Danemark avec Heribert Picht (voir Nuopponen et al. 2000), Gert Engel (voir Grinsted, Nistrup Madsen 1994), Henning Bergenholtz ${ }^{3}$, et en Finlande avec Christer Laurén ${ }^{4}$, Nina Pilke ${ }^{5}$, Anita Nuopponen ${ }^{6}$ en particulier. Les pays de langue néerlandaise, en revanche, ne sont pas inclus, car les thèmes de recherche, ainsi que la langue, y sont depuis longtemps ceux du monde anglo-saxon.

\section{Méthode}

6 La méthode adoptée pour préparer ce panorama est simple. Le point de départ est un dépouillement intégral des dix dernières années des périodiques spécialisés : Lebende Sprachen, Fachsprache pour les pays de langue allemande, Hermes et Sprint pour la Scandinavie, suivi d'une analyse des derniers colloques, surtout European LSP Symposium, mais aussi TKE, TAMA (voir la rubrique Collections et périodiques en fin d'article). Les monographies, qui sont en grand nombre, n'ont pas fait l'objet d'une analyse systématique ; la sélection présentée ici est forcément personnelle. Il convient de signaler, toutefois, que de nombreuses thèses allemandes sont désormais disponibles en ligne, ce qui permet une consultation immédiate. On déplore en revanche que la plupart des monographies signalées ici, ainsi que les périodiques, ne soient pas 
répertoriés sur SUDOC (Système universitaire de documentation, catalogue collectif des bibliothèques universitaires françaises <www.sudoc.abes.fr/>), indication supplémentaire de l'inadéquation des bibliothèques françaises pour les besoins de la recherche en sciences humaines.

\section{Caractéristiques}

\subsection{Quelques points communs}

7 Avant d'aborder les spécificités de la recherche germanique, il convient de rappeler quelques convergences. Dans l'ensemble, on peut dire que l'on relève de nombreux thèmes communs aux recherches en LSP des deux côtés du Rhin. Le terrain d'entente est large. La linguistique textuelle, pratiquée avec des variations nationales, figure comme outil indispensable à bien des études. De même, les études de genres (déroulement d'une communication à un colloque international, structure de l'article de recherche, modes d'emploi,...) sont aussi souvent pratiquées en Allemagne qu'en France. On relève également la même répartition en domaines thématiques : langues juridiques, scientifiques, économiques, etc. Au-delà des questions purement linguistiques, on signale une même préoccupation pour des problèmes de conceptualisation véhiculée par les LSP. À ce propos, on peut signaler un intérêt commun pour la métaphore telle qu'elle est exploitée dans les discours spécialisés. La plupart des francophones qui s'intéressent à ce sujet connaissent déjà le forum électronique metaphorik.de, qui comporte parfois des contributions sur les LSP, mais la tradition allemande dans l'étude des métaphores est ancienne. Nous abordons ce thème ci-dessous. Tous ces sujets communs sont importants, mais l'essentiel est ailleurs : le point de rencontre le plus évident pour tous ceux qui s'intéressent aux LSP est l'intérêt pour la didactique, qui est sans doute sa principale application. La différence serait surtout une question de degré, car cet intérêt semble moins prépondérant dans les pays de langue allemande, et les autres sujets de recherche plus variés.

Rappelons également que l'approche germanique n'a rien de monolithique. Bien au contraire, on assiste à de grands débats, à des luttes d'influence, à des schismes, par exemple entre lexicographes et terminologues. En ce qui concerne ce dernier cas, la différence entre les deux cultures est sans doute due au fait que la lexicographie et la terminologie ne font pas partie en France du paysage des LSP. On constate également une différence entre le degré d'influence qu'a la recherche en langue anglaise et la prise en compte variable des thèmes spécifiquement allemands. On se demande si la jeune génération allemande ne s'intègre pas davantage aux recherches planétaires, tandis que les plus anciens maintiennent le lien avec la recherche propre aux pays du Nord. Liebert (2002), par exemple, tient davantage compte des traditions allemandes que les plus jeunes, comme Drewer (2003). On signale également des différences de réaction par rapport à la question de savoir comment remédier aux insuffisances théoriques en LSP, sujet auquel nous revenons dans le cadre de l'évaluation de la recherche. 


\subsection{Quelques spécificités}

\subsubsection{Une étude systématique placée au sein de la linguistique}

9 La première caractéristique des études des langues de spécialité dans les pays à tradition germanique est la position qu'elles occupent en tant qu'application de la linguistique. Le manuel classique de LSP (Fluck 1996: 10) souligne l'importance de l'ancrage linguistique pour ces études, mais il signale en même temps le rôle que les langues de spécialité sont appelées à jouer en tant que branche de la linguistique ou de la linguistique appliquée. Cette vision, qui se veut globale, fait partie de l'héritage des ambitions des linguistes de l'école de Prague, qui se donnaient pour tâche de rendre compte du fonctionnement de la communication en général, ainsi que de la communication spécialisée, grâce à l'application systématique d'outils développés dans des cadres aussi variés que ceux de la phonologie ou de la norme littéraire. L'empreinte de cette tradition est visible dans les études de LSP telles qu'elles se pratiquent encore en Allemagne. En effet, elles se caractérisent par le recours à une analyse systématique, qui présente à l'examen chaque paramètre du système.

Comme exemple de cette tradition, et de son insistance sur une démarche systématique, nous prenons l'analyse que fait Klaus-Dieter Baumann (2005) des normes qui opèrent dans la communication spécialisée, et dont l'ambition a surpris Philip Shaw. Visiblement nous touchons du doigt à une spécificité du programme de recherche germanique: éclairer la structure et les modes de pensée des "spécialismes ». Baumann fait appel aux préceptes de l'école de Prague développés en particulier par Havránek $(1929,1932)$, désormais accessibles grâce aux publications de Kocourek (2001: 272-4) : fonctions pratiques et théoriques de la communication spécialisée, rôle de la convention, codification. Il s'agit d'une application de la théorisation de la norme littéraire, établie grâce à des études inductives-empiriques.

11 Nous présentons sous forme de tableau les principaux critères dégagés dans l'étude de Baumann (voir tableau 1). Il convient de souligner que cet auteur, fidèle à la tradition allemande qui ne vise pas spécialement à faciliter l'accès à la connaissance spécialisée (Clyne $1987: 66$ ), n'inclut aucun tableau; celui qui figure ci-dessous, qui n'engage que nous-même, a été constitué à la lecture de l'article cité. Il a le mérite, nous semble-t-il, de souligner l'approche systématique d'un sujet fondamental de la communication spécialisée (la manifestation des normes) et de présenter une grille d'analyse qui permet d'orienter des recherches futures. On peut imaginer ce type de tableau comme ébauche d'un programme de recherche à réaliser.

Tableau 1. Manifestations des normes de la communication spécialisée (d'après Baumann 2005)

\begin{tabular}{|l|l|l|}
\hline $\begin{array}{l}\text { Niveau } \\
\text { normes }\end{array}$ & des & Exprimées par \\
\hline $\begin{array}{l}\text { Culturel (culture } \\
\text { spécifique) }\end{array}$ & $\begin{array}{l}\text { Normes culturelles de la } \\
\text { communauté }\end{array}$ & $\begin{array}{l}\text { Nécrologies; revue d'entreprise; } \\
\text { compte rendu d'expérience } \\
\text { Aspects culturels des méthodes de } \\
\text { recherche scientifique Bactériologie } \\
\text { des Allemands/génie génétique des } \\
\text { Américains... }\end{array}$ \\
\hline
\end{tabular}




\begin{tabular}{|c|c|c|}
\hline Social & $\begin{array}{l}\text { a) statut social des interlocuteurs } \\
\text { b) orientation politique/ } \\
\text { Weltanschauung } \\
\text { c) systèmes des valeurs sociales, } \\
\text { mode de pensée } \\
\text { d) degré de connaissance des } \\
\text { interlocuteurs } \\
\text { e) degré de spécialisation/caractère } \\
\text { public de l'échange }\end{array}$ & $\begin{array}{l}\text { Normes par rapport aux attentes du } \\
\text { public visé dans l'acte de } \\
\text { communication } \\
\text { - normes d'emploi de connecteurs dans } \\
\text { les textes didactiques, de vulgarisation }\end{array}$ \\
\hline $\begin{array}{l}\text { Situationnel/ } \\
\text { professionnel }\end{array}$ & $\begin{array}{l}\text { Processus de communication réglée : } \\
\text { armée, administration, justice, } \\
\text { médecine }\end{array}$ & $\begin{array}{l}\text { Normes de communication militaire, } \\
\text { juridique, médicale... }\end{array}$ \\
\hline Cognitif & $\begin{array}{l}\text { Les modes de pensée partagés, ex. } \\
\text { sciences exactes, sciences humaines }\end{array}$ & $\begin{array}{l}\text { À l'intérieur d'un groupe : les } \\
\text { physiciens s'entendent mieux entre eux } \\
\text { qu'avec des philologues }\end{array}$ \\
\hline Disciplinaire & $\begin{array}{l}\text { Différences entre sciences humaines } \\
\text { et sciences naturelles }\end{array}$ & \\
\hline Fonctionnel & $\begin{array}{l}\text { Par rapport aux connaissances } \\
\text { partagées }\end{array}$ & \\
\hline $\begin{array}{l}\text { Morpho- } \\
\text { syntaxique }\end{array}$ & $\begin{array}{l}\text { Moyens exploités pour atteindre la } \\
\text { cohérence/cohésion }\end{array}$ & \\
\hline Stylistique & & $\begin{array}{l}\text { Emploi des citations, déroulement du } \\
\text { texte }\end{array}$ \\
\hline $\begin{array}{l}\text { Lexical/ } \\
\text { sémantique }\end{array}$ & $\begin{array}{l}\text { Rôle de la terminologie, accès aux } \\
\text { définitions spécifiques }\end{array}$ & \\
\hline Orthographique & Sigles, acronymes, etc. & \\
\hline $\begin{array}{l}\text { Phonétique/ } \\
\text { phonologique }\end{array}$ & & \\
\hline
\end{tabular}

12 Comme exemple de lecture de la grille, on peut gloser par exemple sur le caractère du critère social, qui, pour Baumann, est bien plus vaste que le degré de connaissance que partagent les interlocuteurs et que le degré de spécialisation du sujet en question, car il englobe aussi leur statut social, l'orientation politique etc., qui influe également sur les normes qui seront - inconsciemment en général - appliquées. Le plus souvent, Baumann ne donne pas d'exemple d'un critère ou de son application, mais le lecteur peut le faire. Par exemple, au niveau phonétique, le débit de la météo marine se prête parfaitement à une analyse qui tienne compte de ses spécificités, de ses normes. Il ne faut pas oublier non plus que la grille représente une simplification par rapport à l'article. On a gommé certains aspects pour mieux en faire ressortir d'autres, comme 
par exemple le caractère implicite ou explicite des normes, ainsi que d'autres critères transversaux.

\subsubsection{L'équipement de la recherche}

La recherche en LSP est donc caractérisée dans les pays de tradition germanique par une très grande attention portée au caractère systématique de toute analyse et à la nécessité d'élaborer une méthodologie très explicite. Elle a, entre autres conséquences, l'avantage de produire une vaste documentation qui est tout aussi structurée. Signalons d'abord le rôle que joue - pour l'ensemble de la linguistique d'ailleurs - de grandes encyclopédies Handbücher zur Sprach und Kommunikationsforschung (Walter de Gruyter), qui n'ont pas d'équivalent au monde. Pour la langue de spécialité, les volumes sont intitulés Fachsprachen/Languages for Special Purposes (Hoffmann, Kalverkämper \& Wiegand, dir. $(1998,1999))$. Ils comportent plus de trois milles pages et 273 articles; sur les 150 articles du premier volume, 149 sont en allemand. En outre, les autres volumes de la série comportent également des articles qui intéressent les langues de spécialité, en particulier ceux consacrés au lexique (Lexikologie/Lexicology 2002) et aux dictionnaires (Wörterbücher/Dictionnaires/Dictionaries 1989-1991). Les périodiques sont également systématiques et pluridisciplinaires: Fachsprache généraliste, s'intéresse autant à l'allemand qu'aux langues étrangères, Lebende Sprachen, est nettement orienté vers la traduction spécialisée. Les éditeurs proposent par ailleurs des collections où les langues de spécialité figurent en bonne place. La plus importante est sans doute le Forum für Fachsprachen-Forschung, chez Gunter Narr, qui en 2007 compte 82 titres. À titre de comparaison, le même éditeur propose 74 publications dans le domaine de l'anglistique. Ces publications comportent des manuels : celui de Fluck (1996), déjà mentionné, fait figure de classique, car il en est à sa cinquième édition depuis 1976, mais on signale aussi beaucoup de recherches ponctuelles, généralement issues de thèses particulièrement réussies. Celles-ci présentent encore des "portraits" de langues de spécialité données, comme par exemple Hänchen (2002), sur le français du marketing, mais aussi sur le rôle de la métaphore dans la recherche et la vulgarisation scientifiques (Drewer 2003), parmi beaucoup d'autres.

Dans la grande majorité des cas, surtout dans les monographies, on relève une insistance sur la nécessité d'une assise théorique et méthodologique. Celle-ci aboutit inévitablement à la constatation que les langues de spécialité ont fait l'objet de beaucoup de travail utile mais qu'elles sont encore insuffisamment théorisées. Une des meilleures expressions de ce type de bilan se trouve précisément dans l'encyclopédie, lorsque deux des trois rédacteurs, Hoffmann et Kalverkämper (1998), présentent les réalisations pratiques qui sont effectivement nombreuses, qui répondent à des besoins précis et qui participent d'une systématisation des données et d'une prise de conscience des problèmes posés. La tendance à élargir le domaine de la recherche, désormais étendue à la communication spécialisée plutôt qu'aux langues de spécialité, est évaluée de façon positive, mais les deux rédacteurs sont obligés de constater, malgré le chemin parcouru, qu'il reste un " déficit douloureux » en matière de théorie des langues de spécialité ${ }^{7}$, constatation qui trouve des échos auprès de certains spécialistes francophones, par exemple Yves Gambier (1998), lui-même professeur de traduction en Finlande. 


\subsubsection{L'importance de l'histoire} sur une connaissance approfondie de nombreux paramètres des langues de spécialité, indication $\mathrm{du}$ respect du critère de l'érudition mentionnée en introduction. Elle s'exprime de nombreuses façons, mais l'une des plus importantes est le lien avec le passé. L'importance de l'école de Prague a déjà été mentionnée: les travaux de Havránek $(1929,1932)$ portant essentiellement sur les fonctions pratiques et théoriques de la communication spécialisée, le rôle de la convention, de la codification, trouve encore des échos dans les travaux actuels, comme nous l'avons vu avec Baumann. Cette insistance sur les fonctions de la communication spécialisée a débouché sur l'élaboration d'une Wirtschaftslinguistik (linguistique du langage de l'économie, voir Picht 1998) de la période de l'entre deux guerres, qui est reconnue comme l'ancêtre direct des études de langue de spécialité ${ }^{8}$. allemande, celle de l'onomasiologie, développée sous le nom d'une revue, fondée à Heidelberg en 1909, Wörter und Sachen, et connue surtout par les publications de Hugo Schuchardt, Sachen und Wörter de 1912. Elle a été déterminante dans la formulation d'une première ébauche d'une théorie sur le lexique spécialisé sous la forme de la thèse d'Eugen Wüster (1932), qui débouchera plus tard sur une théorie de la terminologie. L'innovation que comportait cette thèse a été rappelée un quart de siècle plus tard (Weisgerber 1958), car elle ouvrait la voie à l'étude de cette partie de la langue que les hommes construisent en réponse aux besoins de la science et des technologies. Les études des métaphores, déjà mentionnées, puisent également dans une riche tradition allemande, celle des Bildfelder, les champs d'images, qui sont encore exploités par des linguistes tels que Liebert (2002).

L'importance de l'histoire de la linguistique appliquée a son pendant dans le développement des études sur l'histoire de la langue, y compris les langues de spécialité. L'encyclopédie (Hoffmann et al. 1998, 1999) en comporte un grand nombre dans différentes langues, y compris l'anglais, et on compte une douzaine d'articles sur l'anglais de spécialité du XIXe et du XXe siècles, allant de la chimie (Crosland 1999) à la médecine (French 1999) en passant par les langages linguistiques, littéraires et pédagogiques (Walmsley 1999, Salmon 1999).

es études commencent seulement à puiser dans la méthodologie de l'étude diachronique des langues de spécialité proposée par Walter von Hahn (1983) qui préconise une méthodologie réfléchie des références croisées selon le modèle de la philosophie de l'histoire. À un moment où la terminologie diachronique commence timidement à faire son apparition en France, il serait opportun sinon de traduire au moins de présenter les grandes lignes de la méthodologie. En Allemagne, les études de ce type sont toujours assez rares, mais on peut signaler une thèse remarquable sur l'histoire de la création des concepts mathématiques au XIX ${ }^{e}$ siècle et de leur circulation dans les principales langues européennes (Becker 2005).

Walter von Hahn est également connu pour avoir impulsé des études sur le caractère vague des langues de spécialité, prenant ainsi le contre-pied de ceux qui prétendaient qu'elles se caractérisent par la précision. C'est un aspect qui a renouvelé en particulier l'étude des discours juridiques et administratifs. 


\subsubsection{L'importance du lexique}

20 L'une des grandes différences entre l'étude des langues de spécialité dans les pays anglophones et francophones d'un côté et germanophones de l'autre est la prise en compte de la composante lexicale. Cette différence s'explique en partie du fait du développement relativement tardif des études de LSP en dehors de la Germanophonie, à une époque où le lexique n'occupait plus le devant de la scène. Comme ailleurs, l'importance du lexique commence à être relativisée dans les pays de langue allemande, comme le titre d'un ouvrage de L. Hoffmann de 1988 le laisse penser : Vom Fachwort zum Fachtext - Du terme au texte spécialisé. Néanmoins il continue de faire l'objet de recherches très variées.

21 On peut distinguer au moins trois grands courants concernant le lexique. Le premier est sa présentation sous forme de dictionnaires. La lexicographie est plus qu'une pratique, c'est une méthodologie et, à partir des observations tirées de la pratique, elle commence à se constituer en théorie. La lexicographie théorique doit beaucoup à $\mathrm{H}$. Wiegand $(1987,1989)$, qui a l'ambition d'élaborer une théorie autonome et générale de la lexicographie, comme de la métalexicographie, basée sur les besoins des usagers. Le point de départ de la lexicographie fonctionnelle est la déduction a priori des besoins. Bergenholtz et Tarp (2003), les premiers auteurs à avoir conçu un manuel de lexicographie spécialisée (Bergenholtz \& Tarp 1995), élaborent une théorie modulaire, dérivée de la théorie des fonctions lexicographiques, dégroupées selon les besoins les plus variés, par exemple pour les différents types d'apprenants. Ces théories s'appliquent aussi bien aux besoins lexicographiques de type langagier que de type factuel, et Bergenholtz est allé jusqu'à collaborer avec un biologiste pour faire un dictionnaire du génie génétique adapté aux besoins de l'utilisateur moyen (Kaufmann \& Bergenholtz 1992).

Le second courant est l'étude du lexique en soi, souvent orientée vers sa manifestation principale en LSP, à savoir la terminologie, qui en représente le troisième courant. Fluck (1996 : 12), dans le manuel désormais classique de LSP, l'exprime ainsi :

Die Besonderheit der Fachsprache hingegen - auch darüber herrscht weitgehende

Einigkeit - liegt einmal in ihrem spezifiellen, auf die Bedürfnisse des jeweiligen Faches abgestimmten Wortschatz, dessen Übergänge zur Gemeinsprache fliessend sind und der auch gemeinsprachliche und allgemeinverständliche Wörter enthält. (Fluck $1996: 12)^{9}$

23 Tout comme le mot Fachsprache n'est pas un terme (ou pas de façon primaire), une distinction est souvent observée en allemand entre "Fachwort vs Terminus: toute expression d'une langue de spécialité vs désignation d'un concept susceptible d'une définition conventionnelle » (Fluck 1996: 47, qui cite à ce propos le linguiste pragois Beneš).

Les lexicographes et les terminologues germaniques avaient souvent des relations houleuses, mais entre la terminologie et la lexicologie, c'est l'entente cordiale, comme on peut le relever dans le volume (l'un des rares parus actuellement) sur la lexicologie, où l'on relève plusieurs articles de fond sur des questions de vocabulaire spécialisé. L'un des plus intéressants à nos yeux est celui de Kretzenbacher (2002) qui examine le caractère systématique des vocabulaires scientifiques par le biais de grilles de lecture. Le tableau 2 résume les thèses de Kretzenbacher. 
Tableau 2. Tendances vers l'ouverture ou vers la fermeture des vocabulaires scientifiques (d'après Kretzenbacher 2002)

\begin{tabular}{|l|l|l|}
\hline Dimension & Tendance à la fermeture des systèmes & Tendance à l'ouverture des systèmes \\
\hline Intradisciplinaire & $\begin{array}{l}\text { Proximité de terminologie et } \\
\text { nomenclature dans une discipline } \\
\text { donnée }\end{array}$ & $\begin{array}{l}\text { Définitions moins tranchées : énergie } \\
\text { en physique ; récepteur en biochimie }\end{array}$ \\
\hline Interdisciplinaire & cf. champ en sémantique, physique & $\begin{array}{l}\text { cf. vocabulaire de la philosophie qui } \\
\text { s'emploie dans les sciences humaines; } \\
\text { de la physique en sciences }\end{array}$ \\
\hline $\begin{array}{l}\text { Extra- } \\
\text { disciplinaire }\end{array}$ & $\begin{array}{l}\text { Carninoma cervicis uteri } \\
\text { Zervixkarzinom }\end{array}$ & $\begin{array}{l}\text { Expressions du marketing, du } \\
\text { journalisme pénètrent dans la langue } \\
\text { commune }\end{array}$ \\
\hline Interculturelle & $\begin{array}{l}\text { Tendances vers la normalisation, mais } \\
\text { faux-amis : typhus (angl) }=\text { Fleckfieber } ; \\
\text { Traductions de Freud : Trieb = drive, } \\
\text { instinct }\end{array}$ & $\begin{array}{l}\text { cf. angl (ou fran) Gestalt en psycho ; } \\
\text { eigen- en mathématiques }\end{array}$ \\
\hline
\end{tabular}

Contrairement à la France, où ce type de travail semble désormais délaissé, la lexicologie s'illustre en Allemagne par un grand nombre d'études sur l'anglicisation des vocabulaires, en particulier des vocabulaires spécialisés, à commencer par celui de la physique (Schmitt: 1985), les nouvelles technologies de l'information et de la communication: informatique en général (Chang: 2005), l'Internet (Jansen : 2005), Deutsche Telecom (Wetzler : 2006).

Ces études s'appuient sur une tradition qui légitime l'implication d'un angliciste dans la confection d'un dictionnaire d'anglicismes. C'était le cas de Broder Carstensen et puis d'Ulrich Busse, qui publient entre 1993 et 1996 un dictionnaire allemand des anglicismes, en trois volumes, et de Manfred Görlach, le directeur d'une équipe qui a publié, entre 2001 et 2002, un dictionnaire européen comparatif des anglicismes (Dictionary of European Anglicisms), ainsi qu'une bibliographie analytique et un historique. Le vocabulaire spécialisé joue un rôle important dans ce cadre, car c'est surtout par ce biais que les anglicismes pénètrent dans la langue. Dans les pays scandinaves, la concurrence de l'anglais devient si âpre surtout dans les domaines de pointe en science et en technologie que l'on voit paraitre des études sur la perte de domaines, domaines où la science ne se fait plus dans la langue nationale, comme celle de Pia Jarvad (2001).

La terminologie a des applications directes dans le domaine de la traduction spécialisée, mais elle est également un élément clé dans les recherches en intelligence artificielle et dans le transfert des connaissances. En Allemagne, ainsi qu'en France également, plusieurs linguistes qui travaillent surtout en lexicologie, comme Wolf-Andreas Liebert, spécialiste de la métaphore, enseignent en transfert de connaissances dans un département d'informatique ${ }^{10}$. 


\subsubsection{L'importance de la communication spécialisée} communication multiculturelle, comme Finn Frandsen et Winni Johansen (pour un article en français qui est typique de ce changement d'orientation, voir Frandsen \& Johansen 2001). Autre signe des temps, le bulletin d'information des LSP publié à la Handelshøjskole de Copenhague pour le compte de l'UNESCO, UNESCO ALSED-LSP (Anthropologie et sciences du langage dans le développement éducationnel - langages spécialisés 1977-2000) a changé de titre et d'orientation, pour devenir LSP and Professional Communication. La communication en entreprise est un thème qui est particulièrement développé dans les pages de Fachsprache comme de LSP and Professional Communication, comme dans l'article de Satzger (2005). La qualité de communication est un sous-thème particulièrement riche, développé par Engberg et Göpferich (2003). L'intérêt pour les aspects commerciaux de la communication spécialisée ne s'est pas substitué à celui pour les autres types de communication professionnelle, comme par exemple le courrier des médecins, analysé par Jakob en 2005. Sur le plan strictement scientifique, il est intéressant de signaler l'existence d'une série d'articles et d'entretiens avec des scientifiques qui parlent de leur recherche et de leur langue l'allemand, son usage par rapport à l'anglais, la terminologie des lauréats du prix Nobel Nobelpreis Fachlexikalisch: Peter Christian Aichelburg, Fiorenza Fischer, Mathias Zirm (Fachsprache 28, 2006, 102-109).

\subsubsection{La traduction spécialisée : une optique LSP}

30 Le rôle de la terminologie dans la traduction spécialisée a déjà été mentionné et il n'est pas étonnant de constater qu'elle figure en bonne place dans les études allemandes de traductologie: le périodique Lebende Sprachen est consacré en grande partie aux problèmes de terminologie dans une perspective de traduction, et Fachspache évoque souvent des problèmes plus théoriques, comme celui de l'interférence dans la traduction technique (Horn-Helf 2005). Pour connaître la place de la terminologie dans la théorie de la traductologie, les meilleures études sont sans doute celles de Heidrun Gerzymisch-Arbogast. Elle situe la terminologie à la base de la traduction technique en particulier dans les premiers chapitres de son manuel (Gerzymisch-Arbogast 1996). En France, on fait souvent abstraction de la terminologie tant du côté des Ladmiraliens que de ceux des Séleskovitchistes, sans doute à cause d'une orientation influencée par les interprètes. Or, il est connu que les interprètes n'ont pas du tout les mêmes besoins en terminologie que les traducteurs spécialisés. La terminologie est avant tout un phénomène de l'écrit. Les nombreux écrits de Daniel Gouadec, dont beaucoup sont réunis sur son $\operatorname{site}^{11}$, comme les formations dans le cadre des universités d'automne, 
illustrent bien le rôle déterminant de la terminologie en traduction spécialisée et méritent d'être mieux connus en Allemagne.

31 Chaque pays a sa spécialité en matière de traduction: le Danemark est fortement spécialisé en traduction juridique. Les orientations divergent. Certains suivent une logique lexicographique, comme Sandro Nielsen $(1994)^{12}$, d'autres sont plus éclectiques comme Marianne Garre (1999), dont les travaux en traduction des droits de l'Homme ont alimenté la recherche d'Elsa Pic (2007-2008) sur la définition de l'anglais des droits de l'Homme.

\subsubsection{Liens sciences/société}

Les liens entre les spécialistes de langues de spécialité et la société sont évidents dans les rapports qu'ils entretiennent avec les entreprises (voir aussi Kresta 2003), mais ils prennent également d'autres formes. Les linguistes sont parties prenantes dans la réforme des pratiques de communication administrative, qui va au-delà de la simplification de la langue. L'importance du plurilinguisme dans les sciences fait l'objet d'un débat qui est aussi fourni en Allemagne qu'en France, et qui se fonde sur des études très précises, comme celle de Skudlik (1995). Les valeurs citoyennes sont mises en avant par des linguistes comme Ehlich (2004) mais contestées par d'autres, qui prétendent que la science reste la même, quelle que soit la langue par laquelle elle est véhiculée.

\subsubsection{Vers l'avenir}

33 Quelle direction prennent aujourd'hui les études de langues de spécialité en Allemagne? Il est difficile de prévoir l'avenir, mais, au moment où un troisième tome de Fachsprachen/Languages for Special Purposes est annoncé, on peut revenir à l'analyse des besoins de Hoffmann et Kalverkämper (1998). Ils préconisaient d'abord de mieux faire dialoguer les différents secteurs que comportent les LSP : la terminologie a besoin de mieux communiquer avec les recherches en LSP en général, comme celles-ci doivent mieux prendre en compte la didactique des disciplines. En effet, les liens entre l'enseignement des sciences et les différents codes qui permettent cette communication méritent bien une collaboration que certains ont déjà appelée de leurs vœux (Schaetzen 1992). La stylistique des textes de spécialité a son rôle à jouer dans le cadre de cette collaboration didactique, tout comme pour la formation des rédacteurs techniques. La pertinence sociale est le mot clé dans cette réflexion. Après tout, l'objectif final est l'éducation, et pas seulement la formation. Le rôle des langues dans cet enseignement l'anglais et la langue nationale - mérite également une réflexion approfondie, et plus généralement la domination de l'anglais doit être évaluée dans les différents secteurs.

D'autres collaborations se révèlent tout aussi indispensables: la sémiotique figure en bonne place, car l'étude de la communication implique les interactions entre le langagier et le non langagier, entre l'oralité et l'écriture, entre le multimédia et ce que Hoffmann et Kalverkämper appellent l'internalité - c'est-à-dire les différences engendrées par l'exploitation des différents médias et leur synthèse nécessaire (Hoffmann \& Kalverkämper 1998 : 365), sans oublier bien sûr l'interculturel. Ils prônent une posture synchronique, mais qui tienne compte de la diachronie. 


\section{Pour le GERAS}

Concrètement, que peut faire le GERAS pour favoriser les échanges avec les pays de langue allemande? Un premier pas serait d'élargir la nature des ouvrages analysés dans la rubrique des recensions à des comptes rendus de publications significatives en LSP - de type bref et surtout signalétique pour les ouvrages en anglais et plus circonstancié pour les livres en allemand, de telle sorte que le chercheur angliciste se tienne informé. Par réciprocité, on peut encourager les auteurs d'ASp de proposer le résultat de leurs recherches à des revues, qui s'ouvrent de plus en plus aux publications « internationales». C'est le cas d'Hermes, qui souhaite précisément élargir non seulement son lectorat mais aussi la palette de ses contributeurs.

Plus généralement, le GERAS se doit de continuer d'ouvrir ses publications et ses colloques à tous ceux qui travaillent dans le secteur des langues de spécialité. Les spécialistes $\mathrm{du}$ français sur objectifs spécifiques sont des interlocuteurs particulièrement proches, comme la conférence de $\mathrm{S}$. Moirand et G. Tréguer-Felten l'a bien démontré en 2007, car ils ont non seulement les mêmes préoccupations, mais ils travaillent aussi sur l'autre langue d'interface, le français. En outre, ils ont des liens privilégiés avec les étrangers qui travaillent en LSP, comme le dernier Carnet $d u$ CEDISCOR le montre, en particulier avec l'étude de Bernd Spillner (2006).

Le GERAS pourrait enfin continuer les invitations aux spécialistes européens des LSP, comme Maurizio Gotti (2004). L'essentiel est de poursuivre la politique d'ouverture, nationale et internationale aussi bien que disciplinaire.

\section{BIBLIOGRAPHIE}

Monographies et ouvrages

Baumann, Klaus-Dieter. 2005. „Das komplexe Normensystem der Fachkommunikation“.

Fachsprache 27, 32-47.

Becker, Holger. 2005. Semantische und lexikalische Aspekte der mathematischen Fachsprache des 19. Jahrhunderts, thèse de l'Université d'oldenburg. <http:// docserver.bis.uni-oldenburg.de/ publikationen/dissertation /2006/becsem05/becsem05.html>.

Bergenholtz, Henning \& Sven Tarp. 2002. „Die moderne lexikographische Funktionslehre. Diskussionsbeitrag zu neuen und alten Paradigmen, die Wörterbücher als Gebrauchsgegenstände verstehen“. Lexikographica. Internationales Jahrbuch für Lexikographie 18, 253-263.

Bergenholtz, Henning \& Sven Tarp. 1995. Manual of Specialised Lexicography. The Preparation of Specialised Dictionaries, John Benjamin : Amsterdam, Philadelphia. V. aussi compte rendu de l'auteur de l'édition danoise : Cahiers de lexicologie 66, 186-194.

Bergenholtz, Henning \& Sven Tarp. 2003. “Two opposing theories: On H.E. Wiegand's recent discovery of lexicographic functions". Hermes 31, 171-196. 
Berndt, Rolf. 1999. „The languages of law in England“. In Hoffmann et al. Fachsprachen/Languages for Special Purposes vol. 2, 2485-2494.

Carstensen, Broder \& Ulrich Busse. 1993-1996. Anglizismen-Wörterbuch. Der Einfluss des Englischen auf den deutschen Wortschatz nach 1945. 3 vol. Berlin, 1993-1996.

Chang, Youngick. 2005. Anglizismen in der deutschen Fachsprache der Computertechnik. Frankfort : Peter Lang. Reihe 21, Linguistik vol. 280.

Clyne, Michael. 1987. "Cultural differences in the organization of academic discourse : English and German". Journal of Pragmatics 5, 61-66.

Crosland, Maurice. 1999. "The language of chemistry from the beginnings of alchemy to c. 1800". In Hoffmann et al. Fachsprachen/Languages for Special Purposes vol. 2, 2477-2485.

Ditlevsen, Marianne et al. 2005. "New Trends in LSP - European LSP Symposium in Bergamo August 29 - September 2, 2005” (compte rendu), Fachsprache 27, 174-180.

Ehlich, Konrad. 2004. "The future of German and other non-English languages". In Gart, A and B. Hüppauf (dir.), Globalisation and the Future of German, Berlin : Mouton de Gruyter, 173-184.

Engberg, Jan \& Susanne Göpferich. 2003. Tagungsbericht : „Qualität fachsprachlicher Kommunikation“. Fachsprache 25, 152-153.

Drewer, Petra. 2003. Die kognitive Metapher als Werkzeug des Denkens. Zur Rolle der Analogie bei der Gewinnung und Vermittlung wissenschaftlicher Erkenntnisse Forum für FachsprachenForschung $n^{\circ}$ 62. Gunter Narr Verlag : Tübingen.

Frandsen, Finn \& Winni Johansen. 2001. «Genre, classification et communauté de discours : pour une nouvelle approche à l'étude des communications organisationnelles ». In F. Bernard (dir.), Les recherches en communication organisationnelle : quels concepts? quelles théorisations? I-II. Rennes : Presses universitaires de Rennes.

French, Roger. 1999. "The special language of anatomy in England from the Middle Ages to the 18th century”. In Hoffmann et al. Fachsprachen/Languages for Special Purposes vol. 2, 2472-2477.

Fuck, Hans-Rüdiger. 1996 [1976]. Fachsprache : Einführung und Bibliographie, 5e édition. Francke Verlag : Tübingen.

Gambier, Yves (dir.). 1998. Discours professionnels en français. Frankfurt am Main : Peter Lang Verlag. v. aussi, compte rendu de l'auteur, Terminologies nouvelles 20, 76-77.

Garre, Marianne, 1999. Translation. Legal Concepts in Different Languages. Handelshøjskole i København.

Gerzymisch-Arbogast, Heidrun. 1996. Termini im Kontext. Verfahren zur Erschliessung und Übersetzung der textspezifischen Bedeutung von fachlichen Ausdrücken, Tübingen : Gunter Narr Verlag. Forum für Fachsprachen-Forschung, Band 31.

Görlach, Manfred (dir.). 2001. A Dictionary of European Anglicisms. Oxford : Oxford University Press. Görlach, Manfred (dir.). 2002. English in Europe. Oxford : Oxford University Press.

Görlach, Manfred (dir.). 2002. An Annotated Bibliography of European Anglicisms. Oxford : Oxford University Press.

Gotti, Maurizio. 2004. "Specialised discourse in multilingual and multicultural contexts". ASp 45-46, 5-20. 
Grindsted, Annelise \& Bodil Nistrup Madsen (dir.). 1994. Festskrift til Gert Engel I anledning af hans 70 års fødselsdag, Kolding : Udgivet af Det erhvervssproglige Fakultet, Handelshøjskole Syd.

Hahn, Walther von. 1983. Fachkommunikation. Entwicklung. Linguistische Konzepte. Betriebliche Beispiele. Berlin : de Gruyter.

Hahn, Walter von. 1998. „Vagheit bei der Verwendung von Fachsprachen“. In Hoffmann et al. Fachsprachen/Languages for Special Purposes vol. 1, 378-382.

Hänchen, Regina. 2002. Die französische Marketingsprache. Eine diachrone Untersuchung ihrer Terminologie anhande der Revue française du marketing. Peter Lang Verlag : Francfort. Voir aussi compte rendu de Jan Engberg, LSP and Professional Communication 5/1, 104-108.

Hoffmann, Lothar. 1988. Vom Fachwort zum Fachtext : Beiträge zur Angewandten Linguistik, Tübingen : Gunter Narr Verlag.

Hoffmann, Lothar, Hartwig Kalverkämper \& Herbert Ernst Wiegand (dir.) (avec Christian Galinski \& Werner Hüllen). 1998, 1999. Fachsprachen, Languages for Special Purposes, Ein internationales Handbuch zur Fachsprachenforschung und Terminologiewissenschaft - An International Handbook of Special Language and Terminology Research, Berlin et New York, de Gruyter, coll. Handbücher zur Sprach- und Kommunikationswissenschaft, vol. 14-1, t. 1 vol. 2. V. aussi compte rendu de l'auteur, Meta 2000.

Hoffmann, Lothar \& Hartwig Kalverkämper. 1998. „Forschungsdesiderate und aktuelle Entwicklungstendenzen in der Fachsprachenforschung “ In Hoffmann et al. Fachsprachen/ Languages for Special Purposes vol. 1, 355-372.

Hohnhold, Ingo. 1990. Übersetzungsorientierte Terminologiearbeit : eine Grundlage für Praktiker, Stuttgart : InTra 1. V. aussi compte rendu de l'auteur, Banque des Mots, numéro spécial, 1991, 102-106.

Horn-Helf, Brigitte. 2005. „Interferenzprobleme beim Übersetzung technischer Texte“. Fachsprache 27, 139-158.

Jakob, André. 2005. „So kommt der Patient auf's Papier: Fachtextanalyse klikischer Artzbrief“. Fachsprache 27, 159-172.

Jansen, Silke. 2005. Sprachliches Lehngut im world wide web. Neologismen in der französischen und spanischen Internetterminologie. Tübingen : Gunter Narr Verlag. Tübinger Beiträge zur Linguistik 484.

Jarvad, Pia. 2001. Det danske sprogs status i 1990'erne med scerligt henblik på domcenetab. Dansk Sprognævns skrifter 32. Københavns Universitet.

Kaufmann, Uwe \& Hennig Bergenholtz. 1992. Genteknologisk ordbog, Copenhagen : Gad.

Kaufmann, Uwe \& Hennig Bergenholtz et al. 1998. Encyclopedic Dictionary of Gene Technology, vol. 1, English (with Spanish equivalents) vol. 2, Spanish (with English equivalents). Lugus Libros : Toronto. v. aussi compte rendu de l'auteur, Meta XLV, 2002.

Kocourek, Rostislav. 2001. Essais de linguistique française et anglaise. Mots et termes, sens et textes. Éditions Peeters : Louvain, Paris.

Kresta, Ronald. 2003. “Die Erstellung eines firmenspezifischen Wörterbuches (Deutsch-Englisch/ English-Deutsch)”. Fachsprache 23, 101-119.

Kretzenbacher, Heinz. 2002. „Wissenschaftsbezogene Wortschätze“. In Cruse et al. (dir.), Lexikologie/Lexicology. Berlin : de Gruyter, 919-925. 
Liebert, Wolf-Andreas. 2002. „Bildfelder in synchronischer Perspektive“, In Cruse et al. (dir.), Lexikologie/Lexicology. Berlin : de Gruyter, 771-783.

Moirand, Sophie \& Geneviève Tréguer-Felten. 2007. « Des mots de la langue aux discours spécialisés, des acteurs sociaux à la part culturelle du langage : raisons et conséquences de ces déplacements ». ASp 51-52.

Nickl, Markus. 2001. Gebrauchsanleitungen, Ein Beitrag zur Textsortengeschichte seit 1950. Tübingen : Gunter Narr Verlag. Forum für Fachsprachen-Forschung 52.

Nielsen, Sandro. 1994. The Bilingual LSP Dictionary. Principles and Practice for Legal Language. Tübingen : Gunter Narr Verlag. Forum für Fachsprachen-Forschung Band 24.

Nielsen Sandro. 2006. "Monolingual accounting dictionaries for EFL text production”. Ibèrica 12, 43-64 <http://www.aelfe.org/documents/04-Nielsen.pdf>.

Nuopponen, Anita, Bertha Toft \& Johan Myking (dir.). 2000. I terminologins tjänst.

Festskrift för Heribert Picht på 60-årsdagen, Vaasa. VAASAN YLIOPISTON JULKAISUJA -SELVITYKSIÄ JA RAPORTTEJA.

Perrin, Michel (dir.). 1983. Actes du $4^{\mathrm{e}}$ symposium européen sur les langues de spécialité.

Pratiques d'aujourd'hui et besoins de demain. Bordeaux : DLPV.

Perrin, Michel. 1995. « Les langues de spécialité, facteur de progrès pédagogique » In Budin (dir.), Multilingualism in Specialist Communication, Proceedings of the 10th European LSP Symposium, 29 August - $1^{\text {st }}$ September 1995, Vienne : IITF/Infoterm/TermNet, 47-83.

Pic, Elsa. 2007-08. 7 « Faire de la terminologie en droit». Cahiers du CIEL <http://www.eila.univparis-diderot.fr/recherche/clillac/ciel/cahiers/2007-2008>.

Picht, Heribert. 1998. „Wirtschaftslinguistik. Ein historischer Überblick“. In Hoffmann et al. Fachsprachen/Languages for Special Purposes. Vol 1, 336-341.

Picht, Heribert (dir.). 2006. Modern Approaches to Terminological Theories and Applications, Berlin : Peter Lang.

Salmon, Vivian. 1999. "The development of special registers in English: a historical review”, in Hoffmann et al. Fachsprachen/Languages for Special Purposes. Vol 2, 2502-2511.

Satzger, Axel. 2005. „Unternehmenskommunikation als Gegenstand der angewandten Linguistik“. Fachsprache 27, 82-98.

Schaetzen, Caroline de. 1992. « La barrière du vocabulaire en première candidature de sciences ». La Banque des mots 43, 15-62.

Schmitt, Peter. 1985. Anglizismen in den Fachsprachen. Eine pragmatische Studie am Beispiel der Kerntechnik. Heidelberg : Carl Winter.

Shaw, Philip. 2006. “Text Purpose, Text Effects, Test Power English for Specific Purposes”. The European English Messenger 15/2, 39-43.

Spillner, Bernd. 2006. « Analyse contrastive des textes multimédias : le cas de la nécrologie ». Les Carnets du Cediscor 9, Paris. Presses de la Sorbonne Nouvelle, 75-90.

Walmsley, John. 1999. „English grammatical terminology from the 16th century to the present“, in Hoffmann et al. Fachsprachen/Languages for Special Purposes vol. 2, 2494-2502. 
Weisgerber, Leo. 1958. „Ein Markstein Angewandter Sprachwissenschaft : Begegnung mit Eugen Wüster : Eugen Wüster zum 60 Geburtstag ". Sprachforum 3/2, 92-95 reproduit in Picht, Schmitz (dir.) Terminologie und Wissensordnung. TermNet Publisher : Vienne.

Wetzler, Dagmar. 2006. Mit Hyperspeed ins Internet. Zur Funktion und zum Verständnis von Anglizismen in der Sprache der Werbung der Deutschen Telekom. Frankfurt am Main : Peter Lang. Europäische Hochschuleschriften. Reihe XIV.

Wiegand, Herbert Ernst. 1987. „Zur handlungstheoretischen Grundlegung der Wörterbenutzungsforschung“. Lexicographica 3, 178-227.

Wiegand, Herbert Ernst. 1989. „Der gegenwärtige Status des Lexikographie und ihr Verhältnis zu anderen Disziplinen“. In Hausmann et al Wörterbücher, Dictionaries, Dictionnaires. 1, 246-280.

Wüster, Eugen. [1932] 1970. Internationale Sprachnormung in der Technik besonders in der Elektrotechnik (Die nationale Sprachnormung unde ihre Verallgemeinerung. $3^{\mathrm{e}}$ édition revue et corrigée. Bonn : H. Bouvier und Co.

Collections et Périodiques

La Banque des mots. Revue de terminologie française, Conseil international de la langue française, Paris. <http://www.cilf.org/bm.fr.html> ; voir les numéros spéciaux du Centre de terminologie et de néologie.

European LSP Symposia : le sommaire des précédents colloques est regroupé sur le site <http:// www.dsff-lsp.dk/LSP/prevlsp01.htm>.

$16^{\text {e }}$ symposium <http://nats-www.informatik.uni-hamburg.de/view/LSP07/WebHome>.

Fachsprache, Internationale Zeitschrift für Fachsprachenforschung, -didaktik und Terminologie/ International Journal of LSP, research, didactics, terminology/Revue internationale pour les langues de spécialité, recherche, didactique, terminologie. Vienne, Wilhelm Braumüller.

Hermes - Journal of Language and Communication Studies, Handelshøjskolen i Århus, Århus. <http:// www.asb.dk/research/dissemination/scifijournals/nethermes.aspx>.

Lebende Sprachen, Zeitschrift für fremde Sprachen in Wissenschaft? und Praxis. Berlin : Langenscheidt.

LSP and Professional Communication. Copenhague <http://www.dsff-lsp.dk/UK/LSPjournal.htm>.

Metaphorik<http://www.metaphorik.de/Journal/>.

Sprint, CBS - Fakultetet for sprog, kommunikation og kultur, Handelshøjskolen i København, Copenhague.

TAMA (Terminology in Advanced Management Applications) <http://137.251.109.40/dl2000/ eventdetail.php?\&setchannelid=1\&eventid=81>.

Terminologies nouvelles, revue du Réseau international de néologie et terminologie, devenue en 2000 Les Cahiers du Rifal. La liste des résumés de tous les numéros, ainsi que le contenu des numéros sont disponibles sur le site <http://www.cfwb.be/franca/termin/liste.htm>.

Terminologie et Intelligence artificielle <http://www-test.biomath.jussieu.fr/TIA/> .

$6^{\mathrm{e}}$ rencontres <http://www.univ-rouen.fr/dyalang/tia2005/>.

Colloque $2007<$ http://www-sop.inria.fr/acacia/tia2007/>.

Terminologie et ontologie <http://ontology.univ-savoie.fr/condillac/evenements/sft/>. 
TKE (Terminology and Knowledge Management), Gesellschaft für Terminologie und Wissenstransfer/Association for Terminology and Knowledge Transfer/ Association pour la terminologie et le transfert des connaissances <http://www.uibk.ac.at/gtw-org/tkefr.hlml>.

\section{NOTES}

1. «Terminology studies are important in the forum, although they hardly figure in AngloAmerican ESP studies. A strong German tradition, represented by many articles of the journal Fachsprache, has combined the traditions of Wörter und Sachen with those of teaching commercial and technical German to produce schools which use analysis of special-purpose language to illuminate the structure and thinking of specialisms » (Baumann 2005). «ESP as I describe it here has grown out of the teaching of commercial and technical English, and retains an orientation less to the thought-patterns encoded in language choices than to the purposes and effects of texts, as in the journals English for Specific Purposes and Anglais de spécialité » (Shaw 2005 : 39).

2. Le colloque, European LSP Symposium, Bergame 29 août-2 septembre 2005 : New trends in LSP, comportait les thèmes suivants : terminologie ; didactique ; analyse de texte et de genre en LSP ; traduction; terminologie; didactique; analyse de texte et de genre en LSP; traduction; perspectives diachroniques; LSP et communication multimédia; aspects multilingues et culturels ; aspects linguistiques des textes spécialisés ; aspects cognitifs de textes spécialisés.

3. <http://www.asb.dk/staff/hb>

4. <http://lipas.uwasa.fi/hut/svenska/clauren.html>

5. <nina.pilke@uwasa.fi>.

6. <http://lipas.uwasa.fi/ atn/>

7. « schmerzliches Defizit: die fehlende Ausarbeitung einer Fachsprachentheorie " (Hoffmann \& Kalverkämper 1998 : 359).

8. Contrairement à l'anglais et au français, l'allemand n'a jamais eu à chercher une expression particulière pour désigner l'objet des études, comme «langue de spécialité, langage spécialisé, langue spécialisée »; language for special purposes, Fachwort, Fachsprache ne constituent pas des termes créés pour exprimer un point de vue d'étude: ils préexistaient, et ils ont été terminologisés par les études dont nous parlons ici.

9. La spécificité de la langue de spécialité - sur ce point il existe un important consensus - réside dans le vocabulaire spécifique déterminé d'après les besoins du domaine concerné, dont les transferts vers la langue générale se font couramment et qui comporte des mots de la langue générale et communément compréhensibles. (traduction de l'auteur)

10. En France, des terminologues et des spécialistes d'intelligence artificielle collaborent au sein de Terminologie et Intelligence Artificielle (TIA <http://tia.loria.fr/>, <http://www-sop.inria.fr/ acacia/tia2007/>). Ces deux champs d'application se sont réunis dans les pays de langue allemande au sein de l'Association Terminologie und Wissenstransfer <http://www.id.cbs.dk/ het/gtw/gtw.html>, qui organisait un colloque intitulé Terminology and Knowledge Engineering, qui a fait des publications remarquées, très comparables à celles de TIA, et bien mieux diffusées. Malgré ces réalisations, terminologues et spécialistes d'intelligence artificielle dans les deux aires culturelles semblent moins travailler ensemble: TKE n'a pas organisé de colloque depuis $2005<\mathrm{http}$ ://www.id.cbs.dk/ het/gtw/gtw.html>. Pourtant, les projets communs ne manquent pas, comme l'intérêt actuel pour les ontologies, qui permettent d'espérer de nouvelles collaborations, comme au colloque Terminologie et Ontologie <http://ontology.univsavoie.fr/condillac/evenements/sft/> organisé à l'initiative de Christophe Roche et de la Société française de terminologie à Chambéry en 2007. Le divorce ici est moins entre aires culturelles française et germanique qu'entre pédagogues des langues et terminologues.

11. <http://www.gouadec.net/indexDG.html> 
12. Son article récent sur l'apport d'un dictionnaire de LSP - ici dans le domaine de la comptabilité - apporte à l'encodage en langue étrangère, ici en anglais <http://www.aelfe.org/ documents/04-Nielsen.pdf>

\section{RÉSUMÉS}

La recherche menée en langues de spécialité dans les pays où l'allemand joue un rôle véhiculaire est caractérisée par l'importance accordée aux modèles théoriques et au traitement systématique des données relevées. La gamme de sujets abordés est également vaste: elle englobe non seulement des aspects didactiques, mais aussi des questions de terminologie et de conceptualisation, de traduction spécialisée et de la théorie de la communication, sans oublier des préoccupations plus générales telles que la responsabilité sociale des chercheurs en langues de spécialité. Cet article peut être considéré comme une bibliographie commentée d'une partie de ces recherches.

Research in languages for special purposes in countries using German as a vehicular language is characterised by a search for both theoretical adequacy and systematic investigation. The range of subjects investigated also tends to be broad, taking into account not only teaching perspectives, but also issues of terminology and conceptualisation, specialised translation and communication theory, without forgetting the responsibility researchers in the LSP field have to society in general. This article may be seen as a commented bibliography of some of the research in these countries.

\section{INDEX}

Mots-clés : colloque de langue de spécialité, langue de spécialité, recherche en allemand, revue Keywords : German research, language for specific purposes, LSP, LSP conference, journal

\section{AUTEUR}

\section{JOHN HUMBLEY}

John Humbley est professeur à l'UFR des Études interculturelles de langues appliquées de l'Université Paris Diderot, où il enseigne dans les formations Industries de la langue et traduction spécialisée et Conception de documentation multilingue et multimédia. Il est membre du laboratoire Lexiques, dictionnaires, informatique, UMR 7187, où il dirige avec Jean-François Sablayrolles la revue Neologica (Éditions Garnier). John.Humbley@eila.jussieu.fr 